\title{
The expected imprint of flux rope geometry on suprathermal electrons in magnetic clouds
}

\author{
M. J. Owens ${ }^{1}$, N. U. Crooker ${ }^{2}$, and T. S. Horbury ${ }^{1}$ \\ ${ }^{1}$ Space and Atmospheric Physics, The Blackett Laboratory, Imperial College London, Prince Consort Road, \\ London SW7 2BW, UK \\ ${ }^{2}$ Center for Space Physics, Boston University, Boston MA 02215, USA
}

Received: 1 June 2009 - Revised: 20 August 2009 - Accepted: 14 October 2009 - Published: 26 October 2009

\begin{abstract}
Magnetic clouds are a subset of interplanetary coronal mass ejections characterized by a smooth rotation in the magnetic field direction, which is interpreted as a signature of a magnetic flux rope. Suprathermal electron observations indicate that one or both ends of a magnetic cloud typically remain connected to the Sun as it moves out through the heliosphere. With distance from the axis of the flux rope, out toward its edge, the magnetic field winds more tightly about the axis and electrons must traverse longer magnetic field lines to reach the same heliocentric distance. This increased time of flight allows greater pitch-angle scattering to occur, meaning suprathermal electron pitch-angle distributions should be systematically broader at the edges of the flux rope than at the axis. We model this effect with an analytical magnetic flux rope model and a numerical scheme for suprathermal electron pitch-angle scattering and find that the signature of a magnetic flux rope should be observable with the typical pitch-angle resolution of suprathermal electron data provided ACE's SWEPAM instrument. Evidence of this signature in the observations, however, is weak, possibly because reconnection of magnetic fields within the flux rope acts to intermix flux tubes.
\end{abstract}

Keywords. Interplanetary physics (Interplanetary magnetic fields; Energetic particles) - Solar physics, astrophysics, and astronomy (Flares and mass ejections)

\section{Introduction}

Coronal mass ejections (CMEs) are huge expulsions of solar plasma and magnetic field through the corona and out into the heliosphere, known to be the major cause of severe geomagnetic disturbances (e.g., Cane and Richardson, 2003).

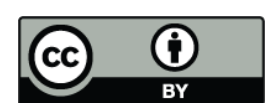

Correspondence to: M. J. Owens (m.owens@imperial.ac.uk)
The interplanetary manifestations of CMEs (ICMEs) provide critical information about their magnetic configuration and orientation, which may prove key in constraining theories of CME initiation as well as aiding our understanding of the evolution of ejecta during their transit from the Sun to $1 \mathrm{AU}$. A variety of signatures are used to identify ICMEs from in situ data, including, but not limited to, low proton temperatures, counterstreaming suprathermal electrons, reduced magnetic field variance and enhanced ion charge states. See Wimmer-Schweingruber et al. (2006) for a more complete review. Magnetic clouds (MCs), a subset of ICMEs comprising somewhere between a quarter to a third of all ejecta (e.g., Cane and Richardson, 2003), are further characterized by a smooth rotation in the magnetic field direction and an enhanced magnetic field magnitude (Burlaga et al., 1981). The field rotation has been attributed to a magnetic fluxrope (MFR, Lundquist, 1950) and commonly modeled as a constant- $\alpha$ force-free MFR (Burlaga, 1988; Lepping et al., 1990), where currents are assumed to be field aligned and $\alpha$ is the constant relating the current density $\boldsymbol{J}$ to the magnetic field vector $\boldsymbol{B}$. This enables single-point, in situ, time series to be interpreted in terms of the large-scale structure of the ejection.

The present study addresses the behavior of suprathermal electrons in magnetic clouds. In general, suprathermal electrons (i.e., $>70 \mathrm{eV}$ ) are of key interest to studies of the solar wind because the field-aligned "strahl" acts as an effective tracer of heliospheric magnetic field topology. A single strahl indicates open magnetic flux (Feldman et al., 1975; Rosenbauer et al., 1977), while counterstreaming electrons (CSEs) often signal the presence of closed magnetic loops with both foot points rooted at the Sun (Gosling et al., 1987). A strong field-aligned strahl is expected and observed in the ambient solar wind near $1 \mathrm{AU}$, as even an initially isotropic distribution near the Sun will undergo pitch-angle focusing due to conservation of magnetic moment in a decreasing magnetic field intensity. Strahl widths at $1 \mathrm{AU}$, however, are much

Published by Copernicus Publications on behalf of the European Geosciences Union. 
broader than would be expected from focusing alone, suggesting significant pitch-angle scattering must occur (e.g., Pilipp et al., 1987). Indeed, with increasing distance from the Sun, pitch-angle scattering becomes increasingly important because the rate of focusing decreases owing to the increasing angle between the spiraling magnetic field direction and intensity gradient (Owens et al., 2008). This results in the strahl width increasing with heliocentric distances (Hammond et al., 1996). For closed magnetic loops in an ICME, the antisunward motion of the loop apex will mean that intransit scattering on the increasingly longer field-line will result in eventual loss of the sunward beam and, hence, loss of the CSE signature, which has important implications for the interpretation of the solar cycle evolution of the heliospheric magnetic field (Owens and Crooker, 2006, 2007).

The flux rope structure of magnetic clouds means the field-line length is shortest at the axis of the MFR, increasing toward the edge as the field winds about the axis. Indirect evidence for the varying length of field lines in magnetic clouds was found in the arrival-time dispersion of solar flare electrons intermittently injected into the October 1995 magnetic cloud (Larson et al., 1997). (Chollet et al., 2007) recently used bursts within energetic particle dispersions within an ICME to infer a jumbled mix of field-line lengths from $\sim 1$ to $3.5 \mathrm{AU}$. While energetic particle events can only be used to calculate field-line length in a very limited number of ICMEs, field-line length should also have an effect on suprathermal electrons, which continually stream away from the Sun along field lines. Since the suprathermal electron time of flight and, hence, pitch-angle scattering time, increases with field-line length, the strahl width should exhibit a characteristic signature as a flux rope convects past an observer at $1 \mathrm{AU}$.

To characterize the expected imprint of flux rope geometry on suprathermal electron observations, we combine two forms of modeling. In Sect. 2, an analytical MFR model is used to calculate the length of magnetic field lines which connect an observer inside an MC to the Sun. In Sect. 3 a numerical model of suprathermal electron evolution is used to estimate the suprathermal electron strahl widths corresponding to the MFR magnetic field line lengths. Finally, in Sect. 5 we look for the predicted variation in strahl width in ACE observations of magnetic clouds.

\section{Magnetic flux rope model}

The classic model for a magnetic cloud-associated flux rope, the constant- $\alpha$ force-free flux rope (Burlaga, 1988; Lepping et al., 1990), assumes the magnetic cloud can locally be approximated as a 2-dimensional cylindrical structure with a circular cross-section. The field is entirely axial at the center of the rope, becoming increasingly poloidal toward the outer edge. It is useful to define a parameter $Y$, the ratio of the distance from the flux rope axis, $r$, to radius of the flux rope, $r_{0}$. The magnetic field of a force-free flux rope is then given by:

$$
\begin{array}{r}
B_{\mathrm{AX}}(Y)=B_{0} J_{0}(\alpha Y) \\
B_{\mathrm{POL}}(Y)= \pm B_{0} J_{1}(\alpha Y)
\end{array}
$$

where $B_{\mathrm{AX}}$ and $B_{\mathrm{POL}}$ are the magnetic field strengths along the axial and poloidal directions, respectively. The poloidal component takes positive or negative values depending on the sense of rotation of the magnetic field about the axis (i.e., the chirality of the flux rope). $J_{0}$ and $J_{1}$ are zero and first order Bessel functions of the first kind, respectively. $\alpha$ is a constant which determines the outer edge of the flux rope. It is normally assumed to be 2.408 , which effectively sets the outer edge of the flux rope at the point where the field first becomes entirely poloidal (Burlaga, 1988; Lepping et al., 1990).

The angle of the magnetic field to the axial direction, $\theta$, is a function of $Y$ :

$\tan \theta=\frac{B_{\mathrm{AX}}}{B_{\mathrm{POL}}}=\frac{J_{0}(\alpha Y)}{J_{1}(\alpha Y)}$

We initially adopt this simple force-free geometry, later modifying the model to incorporate effects which will substantially alter the estimate of field-line length $L$ connecting a 1AU observer to the Sun. The left-hand panel of Fig. 1 defines the basic parameters: The rope is of radius $r_{0}$. At a distance $r$ from the axis, the field takes the form of a helix about the axis, shown as the solid/dashed blue line, and makes an angle $\theta$ to the axis. The field makes one complete revolution about the axis in a height, $H$, along the axis. For an axis of length $L_{\mathrm{AX}}$, the field makes $N$ revolutions (in the example shown, $N \sim 1.8$ ). The right-hand panel shows the curved face of the cylinder unrolled to form a flat plane. The relations between $\theta, H, L_{\mathrm{AX}}, N, r, r_{0}$ and the length of the field line, $L$, are easier to visualize in this way. It can immediately be seen that the length of the field line is:

$L=\sqrt{L_{\mathrm{AX}}^{2}+(2 N \pi r)^{2}}$

and substituting $N H=L_{\mathrm{AX}}$ and $H=2 \pi r / \tan \theta$ gives:

$L=\frac{L_{\mathrm{AX}}}{\cos \theta}$

Thus for a force-free flux rope, the length of the magnetic field line depends only on $\theta$ (which, in turn, is solely a function of $Y$ ) and the length of the axial field line. $L$ is independent of the radius of the flux rope, as the number of revolutions per unit axial length is linearly related to $r$, the distance from the axis.

To estimate $L_{\mathrm{AX}}$, it is necessary to extend the 2dimensional force-free model to a more global configuration. Assuming the flux rope plasma moves radially but the foot points of the flux rope remain connected to the Sun, as suggested by observations (Gosling et al., 1987), the axial field 

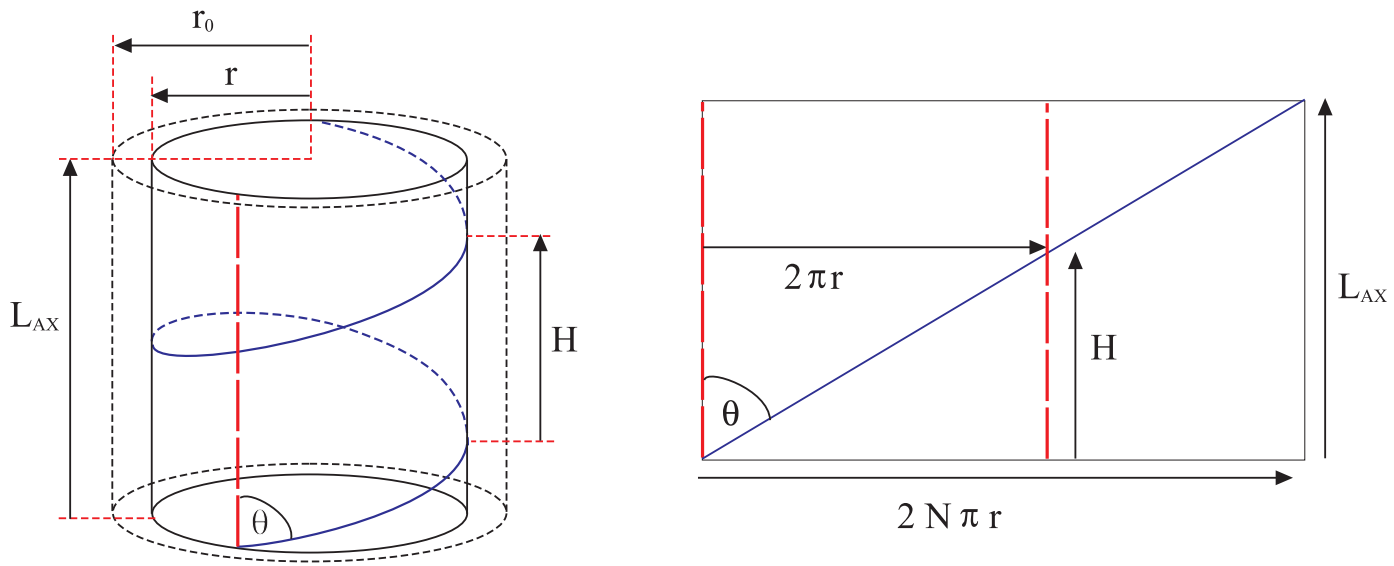

Fig. 1. The left-hand panel shows the cylindrical geometry implied by a force-free flux rope. The rope is of radius $r_{0}$. At a distance $r$ from the axis, the field takes the form of a helix about the axis, shown as the solid/dashed blue line, and makes an angle $\theta$ to the axis. The field makes one complete revolution about the axis in a height, $H$, along the axis. For an axis of length $L_{\mathrm{AX}}$, the field makes $N$ revolutions (in the case shown $N \sim 1.8$ ). The right-hand panel shows the curved face of the cylinder unrolled to form a flat plane. The relations between $\theta, H, L_{\mathrm{AX}}, N, r, r_{0}$ and the length of the field line, $L$ are easier to visualize in this way.

will lie predominantly along the Parker Spiral. Thus at a heliocentric distance $R$, the axis makes an angle $\gamma$ to the radial:

$\gamma=\arctan \left(\frac{\Omega R}{V_{C R}}\right) \cos \lambda$

where $\lambda$ is the heliographic latitude, $\Omega=2 \pi / T_{\mathrm{SID}}$, and $T_{\mathrm{SID}}$ is the sidereal rotation period of the Sun. $L_{\mathrm{AX}}$ is therefore given by:

$L_{\mathrm{AX}}=\int_{0}^{1 \mathrm{AU}} \frac{d R}{\cos \gamma}$

We now include the effect of expansion to allow for axial curvature effects, and later in this section, we incorporate a more realistic cross-sectional topology. Expansion is assumed to be self-similar about the axis, consistent with the linearly declining speed profiles observed within magnetic clouds (Owens et al., 2005). Thus while the axis of the flux rope moves antisunward at a cruise speed $V_{C R}$, the edges of the flux rope move away from the axis at a speed $V_{E X}$. The radius of the flux rope therefore varies with heliocentric distance as:

$r_{0}=\frac{V_{E X} R}{V_{C R}}$

The left panel of Fig. 2 shows a snapshot of the model flux rope using paramters $V_{C R}=400 \mathrm{~km} / \mathrm{s}$ and $V_{E X}=100 \mathrm{~km} / \mathrm{s}$, typical magnetic cloud values (Owens et al., 2005). A heliocentric distance of $1 \mathrm{AU}$ is shown as the black dotted curve, while the Sun is represented by a solid black circle. Only the half of the flux rope which provides the shortest magnetic connection between the Sun and $1 \mathrm{AU}$ is shown. Field lines at $Y=0,0.3,0.6$ and 0.9 are shown by black, blue, green and red lines, respectively. Numerically integrating the distance along each helical path gives field-line lengths of 1.17, 1.23, 1.68 and 5.19 AU, respectively.

The open circles in the right panel of Fig. 2 show the fieldline length between $1 \mathrm{AU}$ and the Sun as a function of distance from the MFR axis. We find the following functional form, shown as the solid black line, adequately describes the model points:

$L[A U]=0.631-0.1176 \tan (-0.288 Y-1.285)$

Finally, we include the effect of MFR cross-sectional elongation in the non-radial direction. Magnetic clouds at $1 \mathrm{AU}$ are known to be highly distorted from the circular crosssection assumed by the force-free approximation. Even if a flux rope has a circular cross-section near the Sun, it will become elongated in the non-radial direction by maintaining a constant angular width as it travels to $1 \mathrm{AU}$ (e.g., Newkirk et al., 1981; McComas et al., 1988; Riley and Crooker, 2004; Owens, 2006). While it is difficult to analytically incorporate this effect into the flux rope model presented here, the increase in field-line length can be approximated by considering the increase in path length around the flux rope cross section. The solid lines in the middle panel of Fig. 2 represent model field non-elongated field lines for $Y=0.3,0.6$ and 0.9 for a force-free flux rope with its axis at $1 \mathrm{AU}$, which has traveled from the Sun at $V_{C R}=400 \mathrm{~km} / \mathrm{s}$ and undergone expansion at $V_{E X}=100 \mathrm{~km} / \mathrm{s}$. Thus at $1 \mathrm{AU}$, the flux rope has a radial width of $2 V_{E X} A U / V_{C R}$. The dashed lines represent the equivalent cross-section for a flux rope with the same characteristic speeds and, hence, radial width at $1 \mathrm{AU}$ as the force-free example but for a MFR which has undergone the kinematic distortion expected from radial propagation (Owens et al., 2006). The increase in field-line length per turn of the magnetic field about the axis is found to be 

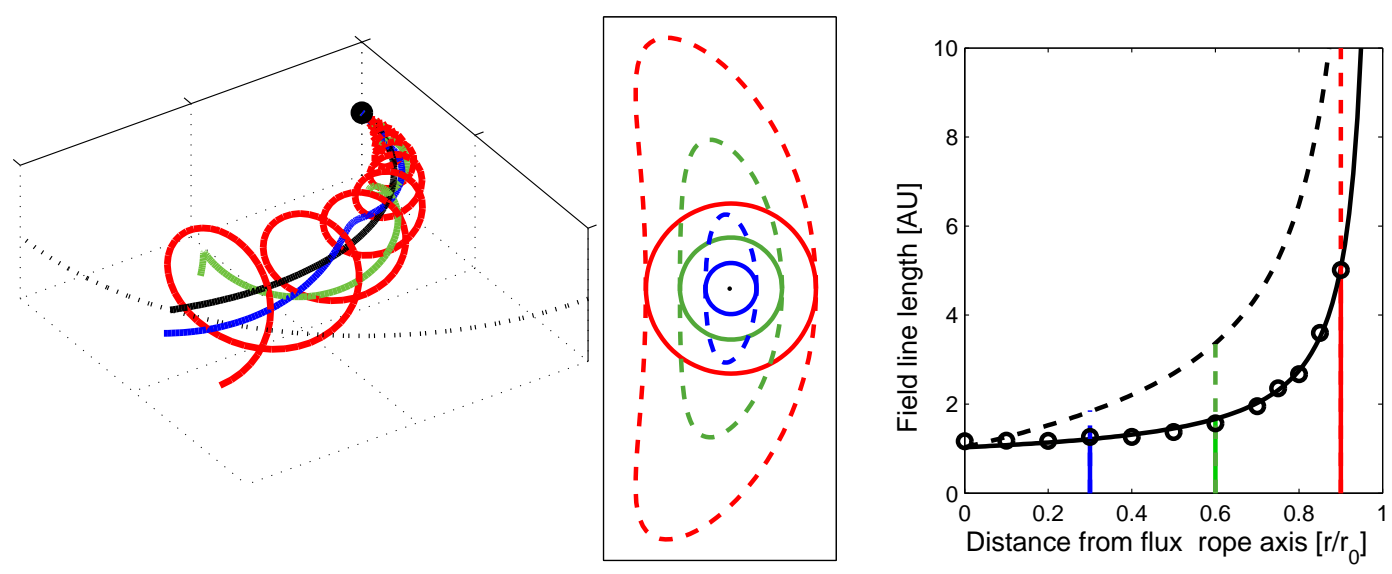

Fig. 2. The left panel shows a snapshot of the model magnetic flux rope. A heliocentric distance of 1 AU is shown as the black dotted curve, while the Sun is represented by a solid black circle. Only the half of the flux rope which provides the shortest magnetic connection between the Sun and $1 \mathrm{AU}$ is shown. Field lines at $Y=0,0.3,0.6$ and 0.9 are shown by black, blue, green and red lines, respectively. Solid lines in the center panel show a cross-section of the flux rope at $1 \mathrm{AU}$, while the dashed lines show how the cross-section is modified by kinematic distortion. The right panel shows the length of the field lines connecting 1-AU to the Sun as a function of distance from the flux rope axis, with solid (dashed) lines indicating a flux rope with a circular (kinematically-distorted) cross section.

$\sim 1.7 Y$. This correction is applied to circular cross section estimates of field line length to approximate the effect of cross-sectional elongation, shown as the dashed line in the right-hand panel of Fig. 2. The best fit is given by:

$$
L[A U]=1.07-0.20 \tan (-0.288 Y-1.285)
$$

\section{Suprathermal electron evolution}

The model of Owens et al. (2008) is used to determine the expected strahl width for a given pitch-angle scattering rate and field-line length. This numerical scheme iteratively solves electron heliocentric distance and pitch-angle, allowing for movement along the magnetic field direction and convection with the bulk solar wind motion. Electrons undergo the competing effects of adiabatic focusing from conservation of magnetic moment and pitch-angle scattering toward an isotropic distribution. The radial evolution of the suprathermal electron strahl width in the fast solar wind can be well matched by this scheme (Owens et al., 2008).

In this study we use a grid of 500 cells in electron pitchangle $(P A)$ space, equally spaced in $\cos P A$. Grid cells are spaced by $0.01 \mathrm{AU}$ in heliocentric distance. Although we are interested in strahl widths at $1 \mathrm{AU}$, the simulation domain extends out to $2 \mathrm{AU}$ to capture the contribution of electrons which are scattered to pitch angles greater than $90^{\circ}$ and thus propagate sunward. A time step of $100 \mathrm{~s}$ and a solar wind speed $\left(V_{S W}\right)$ of $400 \mathrm{~km} / \mathrm{s}$ are used. Electron energy is set at $272 \mathrm{eV}$, as this is the center value of the most commonlyused suprathermal electron energy band for characterizing the strahl (e.g., Anderson et al., 2008).

The model field-line length is adjusted by over- or underwinding a Parker Spiral magnetic field (though the bulk so- lar wind speed experienced by electrons is held constant at $400 \mathrm{~km} / \mathrm{s}$ ). We choose not to use the exact MFR field geometry outlined in the previous section, as it would require extremely high spatial and temporal resolution due to the large gradients in the magnetic field direction, making it computationally prohibitive. Note that the electron model used in this study only accounts for the two major effects: Changing magnetic field strength, which adiabatically focuses electrons, and time of flight, which allows greater pitch-angle scattering to occur. The orientation of the field does not have a direct effect, other than directing electrons into regions of different field strength. Thus while the over/under-wound Parker spiral field will not capture the repeated adiabatic focusing and defocusing of electrons traversing the helical magnetic field of a flux rope, it will capture the net pitchangle focusing and the net amount of scattering experienced by the electrons.

Pitch-angle scattering is performed in an ad-hoc manner, by Gaussian broadening the PA distribution at each time step, pushing the distribution toward isotropy. A broadening factor of $\sigma=0.0014$ applied each second was found to best match the observed strahl width in the fast solar wind (Hammond et al., 1996; Owens et al., 2008). In this study, three levels of scattering are used: The fast solar wind level, taken as "medium" scattering, and "low" and "high" levels of scattering at half and double this value, respectively. The model width at $1 \mathrm{AU}$ is then computed from the model PA distribution using the same fitting procedure as Hammond et al. (1996) and Owens et al. (2008). Suprathermal electron pitch-angle distributions are fit with the following functional form: 


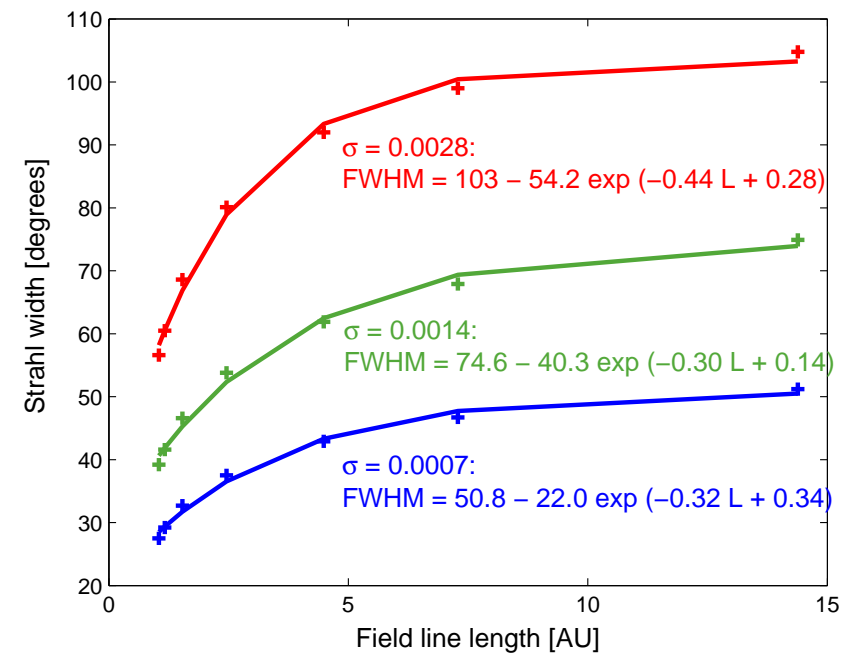

Fig. 3. The effect of varying scattering rate and field-line length on the strahl FWHM at $1 \mathrm{AU}$. As expected, the increased time of flight along longer field lines results in a broader strahl. As $L \rightarrow \infty$, however, the strahl width asymptotes well before isotropy is achieved. This is because the time of flight of the electrons, from the Sun to the observer, has a maximum value of $1 \mathrm{AU} / V_{S W}$ due to the convection of the magnetic field line with the bulk solar wind.

$j(P A)=K_{0}+K_{1} \exp \left[\frac{-P A^{2}}{K_{3}}\right]$

where $j(P A)$ is the differential electron flux at pitch angle $P A, K_{0}$ describes the electron density of the halo and $K_{3}$ determines the width of the strahl (the full width at half maximum is given by $\left.\mathrm{FWHM}=2 \sqrt{(\ln 2)} K_{3}\right) . K_{1}$ is the maximum electron density of the strahl above $K_{0}$.

Figure 3 shows the effect of varying scattering rate and field-line length on the strahl width at $1 \mathrm{AU}$. As expected, the increased time of flight along longer field lines results in a broader strahl. The three levels of scattering are fit with an exponential function, to give the following relations:

$$
\begin{aligned}
& \sigma=0.0007\left[s^{-1}\right]: \\
& \operatorname{FWHM}\left(^{\circ}\right)=51-22 \exp \left[0.34-0.32 L_{A U}\right] \\
& \sigma=0.0014\left[s^{-1}\right]: \\
& \operatorname{FWHM}\left(^{\circ}\right)=75-40 \exp \left[0.14-0.30 L_{A U}\right] \\
& \sigma=0.0028\left[s^{-1}\right]: \\
& \operatorname{FWHM}\left(^{\circ}\right)=103-54 \exp \left[0.28-0.44 L_{A U}\right]
\end{aligned}
$$

where $L_{A U}$ is the field-line length in $\mathrm{AU}$. As $L \rightarrow \infty$, however, the strahl width asymptotes well before isotropy is achieved. This is because the total radial velocity of an electron is given by $V_{S W}+V_{\|} \cos \gamma$, where $V_{\|}$is the electron speed along the magnetic field direction and $\gamma$ is the angle of the field to the radial direction. The first term represents the propagation of magnetic field lines out from the Sun at the bulk solar wind speed. Thus regardless of the field-line length, the maximum time of flight of an anti-sunward propagating electron to a $1-A U$ observer will be $1 \mathrm{AU} / V_{S W}$.

\section{Model time series}

We now combine the MFR and electron scattering models to produce the expected suprathermal electron time series at $1 \mathrm{AU}$. The first step is to generate field-line lengths connecting an observer at $1 \mathrm{AU}$ to the Sun as a magnetic cloud propagates out through the heliosphere. As the expansion speed of magnetic clouds is often a significant fraction of the cruise speed (Owens et al., 2005), we build up a true time series of the model parameters by time evolving past a fixed point at $1 \mathrm{AU}$ rather than take a radial slice through a snapshot of the MFR model.

For this initial time series, the observer is assumed to pass directly through the axis of the flux rope (i.e., through $Y=0$ ), but this is later relaxed when comparing to observations. A cruise (expansion) speed of $400(100) \mathrm{km} / \mathrm{s}$ is used. The top panel of Fig. 4 shows the resulting time series of the magnetic field line length in the flux rope connecting the 1-AU observer to the Sun, with solid (dashed) lines representing a circular cross-section (kinematically distorted) flux rope. Note the logarithmic scale on the y-axis. The second panel shows the result of combining the field-line length with the scattering code to produce a time series of $272 \mathrm{eV}$ electron pitch-angle density at $1 \mathrm{AU}$. This type of plot is commonly used to display suprathermal electron observations. It shows the electron flux at a given energy level, normalized to the maximum and minimum densities at each time step, as a function of pitch angle and time. The strahl width exhibits a clear broad-narrow-broad trend. The third panel shows the computed strahl widths for high (red), medium (green) and low (blue) levels of pitch-angle scattering, for circular crosssection (solid) and kinematically-distorted (dashed) flux rope models. The bottom panel shows the strahl width normalized to the mean strahl width in the event. This normalized width is independent of the scattering rate and only a function of field-line length.

The maximum change in strahl width expected through a MFR, $\triangle F W H M$, is a useful parameter for assessing whether or not the flux rope signature should be observable with a given instrument: If $\triangle \mathrm{FWHM}$ is below the pitch-angle resolution, the effect cannot be measured. Figure 5 shows $\triangle F W H M$ as a function of the closest approach of the observer to the axis of a MFR (i.e., the minimum value of $Y$, often referred to as the "impact parameter"). The color code is the same as in Fig. 4. Very few magnetic clouds are actually encountered "on axis", and thus the minimum value of $Y$ sampled by the observer is normally $>0$. Although $\triangle F W H M$ drops with impact parameter, there is still significant variation in the strahl width even when the observer passes far (e.g., $Y \sim 0.5$ ) from the axis of the flux rope, particularly for higher scattering rates. For extreme glancing encounters of 


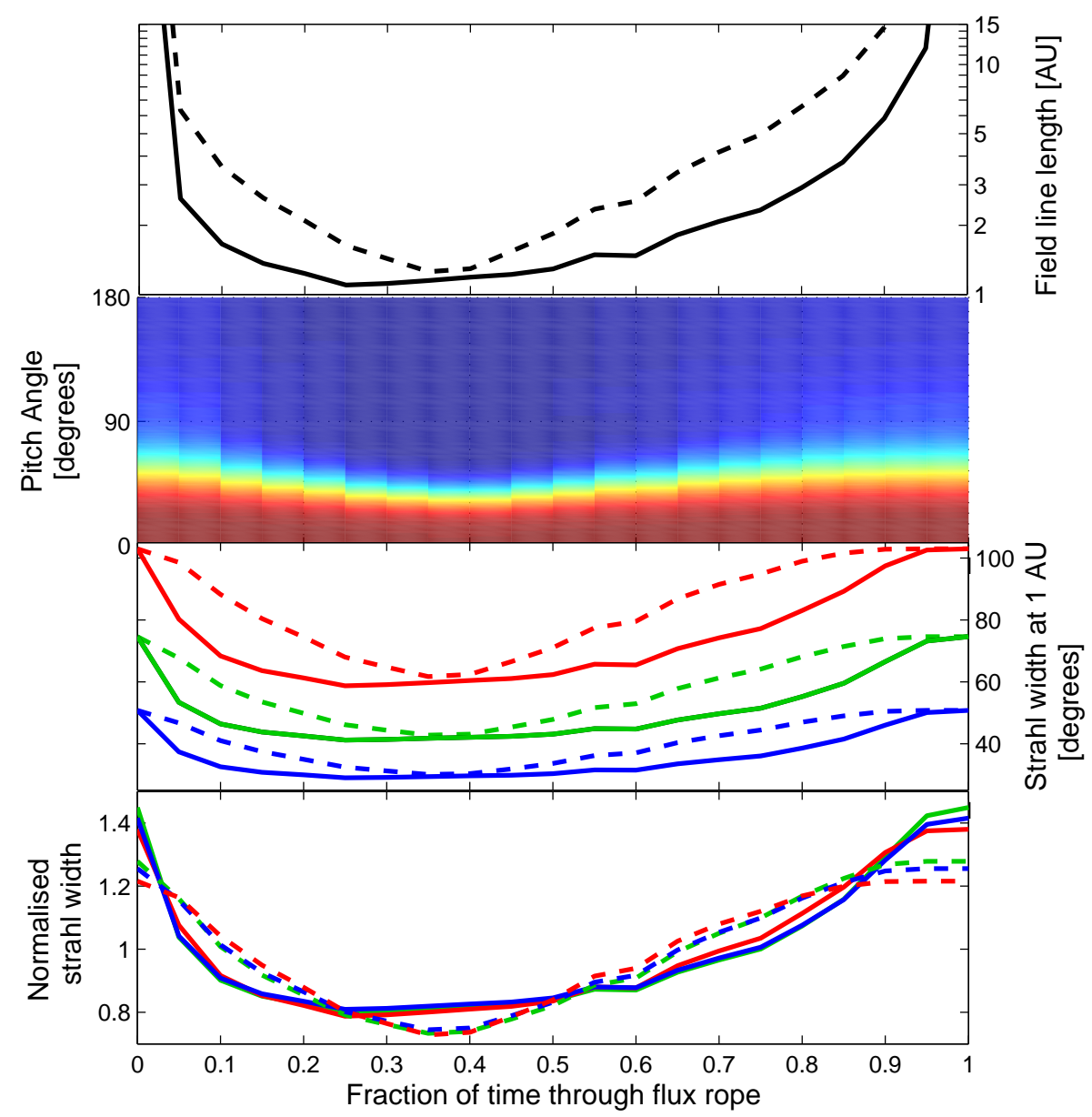

Fig. 4. The model flux rope field-line lengths and expected suprathermal electron signatures. Solid (dashed) lines represent a circular cross-section (kinematically-distorted) flux rope. The top panel shows a time series of field line length connecting an observer to the Sun. Note the logarithmic scale on the y-axis. The second panel shows the normalized 272eV electron pitch-angle density, which exhibits a clear broad-narrow-broad trend through the flux rope. The third panel shows strahl FWHM for high (red), medium (green) and low (blue) levels of pitch-angle scattering. The bottom panel shows the strahl width normalized to the mean width, in the same format.

flux ropes, where the minimum $Y$ intersected by an observing spacecraft is $>0.5$, the field rotation signature will be significantly weaker, and the event is unlikely to be classified as a magnetic cloud.

The SWEPAM instrument (McComas et al., 1998) on the ACE spacecraft collects electron data in approximately $6^{\circ} \times 20^{\circ}$ angular bins. For the standard SWEPAM suprathermal electron data set, the data is then binned into $9^{\circ}$ resolution in pitch-angle space. Figure 5 shows that under most circumstances the difference in strahl width across a flux rope should be more than twice this SWEPAM resolution angle and thus clearly observable.

\section{Observations}

\subsection{Case studies}

In this section we look for the expected suprathermal electron signature of a magnetic flux rope in the ACE SWEPAM (McComas et al., 1998) data. Three magnetic clouds, listed as A, $\mathrm{B}$, and $\mathrm{C}$ in Table 1, have been selected from the Cane and Richardson (2003) ICME list (available at http://www.ssg.sr. unh.edu/mag/ace/ACElists/ICMEtable.html) for their classic form and range of strahl widths.

Figure 6 shows data from Event A. There is a clear rotation in the magnetic field direction, indicative of a magnetic flux rope. This is reflected in the ratios of the eigenvectors in the magnetic field variance directions listed in Table 1 (High ratios, typically $>4$, indicate strong flux rope signatures (e.g., Bothmer and Schwenn, 1998)). Fitting the Owens et al. 
Table 1. Start and end times of three examples of classic magnetic clouds selected from the Cane and Richardson (2003) ICME list. $\lambda_{\mathrm{INT}} / \lambda_{\mathrm{MIN}}$ and $\lambda_{\mathrm{MAX}} / \lambda_{\mathrm{INT}}$ are the ratios of magnetic field variances in the variance directions. $Y$ is the closest approach of the observing spacecraft to the flux rope axis inferred from a flux rope model fit.

\begin{tabular}{cccccc}
\hline Event & Start & End & $\lambda_{\mathrm{INT}} / \lambda_{\mathrm{MIN}}$ & $\lambda_{\mathrm{MAX}} / \lambda_{\mathrm{INT}}$ & $Y$ \\
\hline $\mathrm{A}$ & 20 Aug 1998 06:00 UT & 21 Aug 1998 20:00 UT & 23.4 & 3.75 & 0.12 \\
B & 4 Mar 1998 13:00 UT & 6 Mar 1998 09:00 UT & 15.5 & 4.13 & 0.10 \\
$\mathrm{C}$ & 28 Oct 2000 21:00 UT & 29 Oct 2000 22:00 UT & 3.15 & 7.03 & 0.27 \\
\hline
\end{tabular}

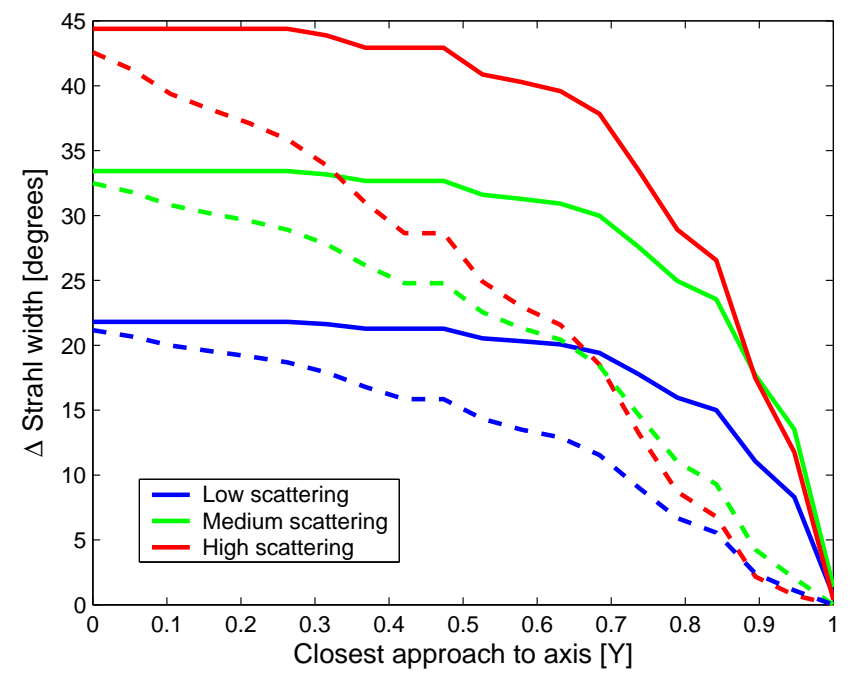

Fig. 5. The maximum expected change in strahl width, a measure of how readily the flux rope signature should be observable, as a function of the closest approach of the observer to the axis of the event (i.e., the minimum value of $Y$, often referred to as the "impact parameter"). The color code is the same as Fig. 4. Although $\triangle F W H M$ drops off with impact parameter, there is significant variation in the strahl width even when the observer passes far (e.g., $Y \sim 0.5$ ) from the axis of the flux rope.

(2006) magnetic cloud model to the observed time series suggests the spacecraft passed close to the axis $(Y=0.12)$. Despite the apparently near-perfect conditions, the expected signature of a dip in suprathermal electron strahl width is not present. This conclusion would not change if alternative cloud boundaries were chosen as the strahl observed immediately before and after the period of magnetic field rotation is narrower, not wider, than inside the event. Note, however, that in the ambient solar wind, the strahl width is generally broader than in the magnetic cloud. As the field-line length is expected to be shorter in the ambient solar wind than in the cloud, pitch-angle scattering must be greatly suppressed in this magnetic cloud compared to ambient conditions. See, however, Event B.

The magnetic field data for Events B and C are not shown, but Table 1 lists the ratios of the eigenvalues in the variance directions to quantify the quality of the flux rope signature.
Also listed are model values of $Y$, the closest approach of the spacecraft to the axis, which are both small.

The top panels of Fig. 7 show the $272 \mathrm{eV}$ suprathermal electron pitch-angle distributions, normalized at each time step, for the three magnetic cloud intervals. For each event, we compute the flux in the $0^{\circ}$ and $180^{\circ}$ strahls (i.e., $K_{1}$ in Eq. 10) throughout the magnetic cloud interval, and define the dominant strahl as that with the highest flux. For Event A the dominant strahl is at $180^{\circ}$ pitch angle, with an order of magnitude higher electron flux than any $0^{\circ}$ strahl. We note, however, that the dominant strahl is not always as easy to define, particularly in events with a strong counterstreaming signature. For Event A, there is a clear broadening of the strahl near the center of the cloud and evidence of counterstreaming near the rear of the cloud. This intermingling of apparently open and closed fields is not uncommon within magnetic clouds (e.g., Crooker et al., 2008). The dominant strahl for Event $\mathrm{B}$ is at $0^{\circ}$ pitch angle. It is much broader than the strahl in Event A. Finally, Event $\mathrm{C}$ has a very narrow strahl at $180^{\circ}$, close to, but above the pitch-angle resolution of the standard SWEPAM suprathermal electron data, with intermittent counterstreaming throughout the cloud. These three events highlight the large event-to-event variability in the suprathermal electron properties of magnetic clouds (see also Anderson et al., 2008).

The black lines in the middle panels of Fig. 7 show the strahl width, computed from the observed pitch-angle distributions using Eq. (10). Solid (dashed) colored lines show the model strahl widths for circular (kinematically-distorted) cross-section flux ropes using the inferred values of $Y$ listed in Table 1. Low, medium and high levels of pitch-angle scattering are shown by the blue, green and red lines, respectively. The bottom panels show the normalized strahl widths in the same format. In all three magnetic clouds, the expected imprint of flux rope geometry on the suprathermal electrons is absent.

\subsection{Statistical survey}

In this section we statistically survey magnetic cloud suprathermal electron profiles. All the events classified as magnetic clouds in the Cane and Richardson (2003) ICME list observed between 1998 and 2007 (74 events) are considered. For each event, the dominant strahl is determined 


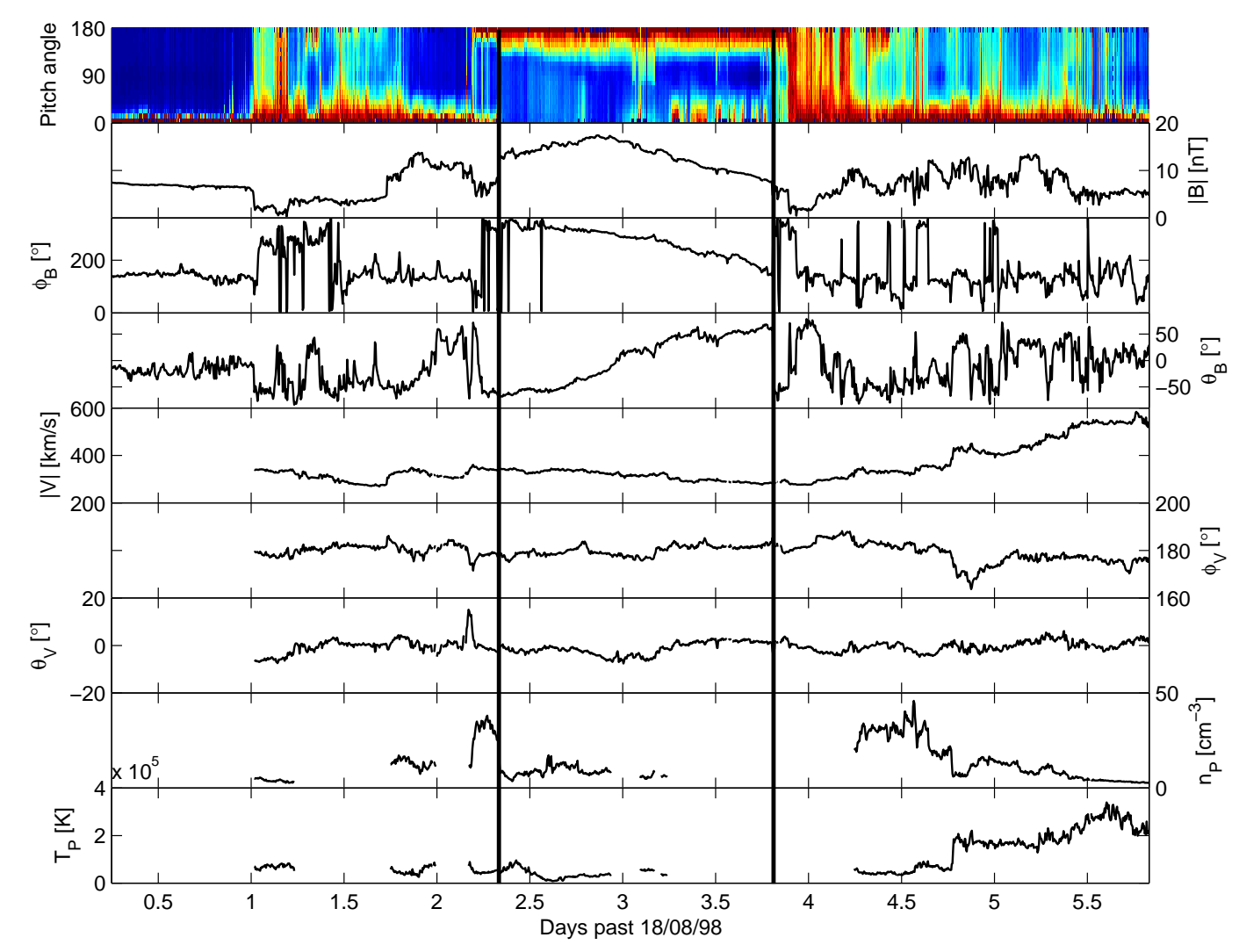

Fig. 6. Event A: A magnetic cloud observed in August 1998. The panels, from top to bottom are: The $272 \mathrm{eV}$ electron pitch-angle distribution, the magnetic field magnitude, the in- and out-of-ecliptic magnetic field angles, the solar wind flow speed, the in- and out-of-ecliptic solar wind flow angles, the proton density and proton temperature. The boundaries of the magnetic cloud are shown by the solid vertical lines. A kinematically-distorted flux rope model fit to the observed magnetic field time series (not shown), suggests the axis of the flux rope passed within $r / r_{0}=0.12$ of ACE at the point of closest approach. The dominant strahl, at $180^{\circ}$ pitch angle, remains $\sim 75^{\circ}$ width throughout the magnetic cloud. Thus the expected suprathermal electron signature of a magnetic flux rope is not present.

within the given magnetic cloud boundaries, and the strahl width is fit using Eq. (10). The left-hand panel of Fig. 8 shows a superposed epoch plot of the strahl width as a function of time, expressed as a fraction of the event's duration. The colored lines show the model predictions in the same format as Fig. 4, assuming on-axis encounters of magnetic clouds. In this view, which covers a wide range of strahl widths to accommodate the full range of scattering constants, the superposed epoch plot shows little variation, and the predicted flux rope signature does not appear to be present. In contrast, the right-hand panel shows the same strahl width data normalized to the mean strahl width within an event. In this format, there is some evidence of the predicted trend in the observations, with an asymmetric dip in strahl width toward the center of magnetic clouds. The trend, however, is much weaker than predicted by the models.

\section{Conclusions}

Inside a magnetic flux rope, the varying pitch of the helical field lines means that their lengths vary substantially between the Sun and an observer. This, in turn, affects the suprathermal electron time of flight and, hence, the pitch-angle scattering time. The strahl width observed as a flux rope convects past a 1-AU observer is therefore expected to exhibit a characteristic variation from a broad strahl at the outer edge that narrows toward the axis and then broadens again toward the opposite edge.

We have combined an analytical magnetic flux rope model with a numerical suprathermal electron scattering code to estimate this expected imprint of flux rope geometry on suprathermal electrons in magnetic clouds. The field-line length is found to increase by more than an order of magnitude from the axis to the edge of the flux rope. The associated variation in strahl width at $1 \mathrm{AU}$, however, is not as extreme because convection of magnetic field lines with 

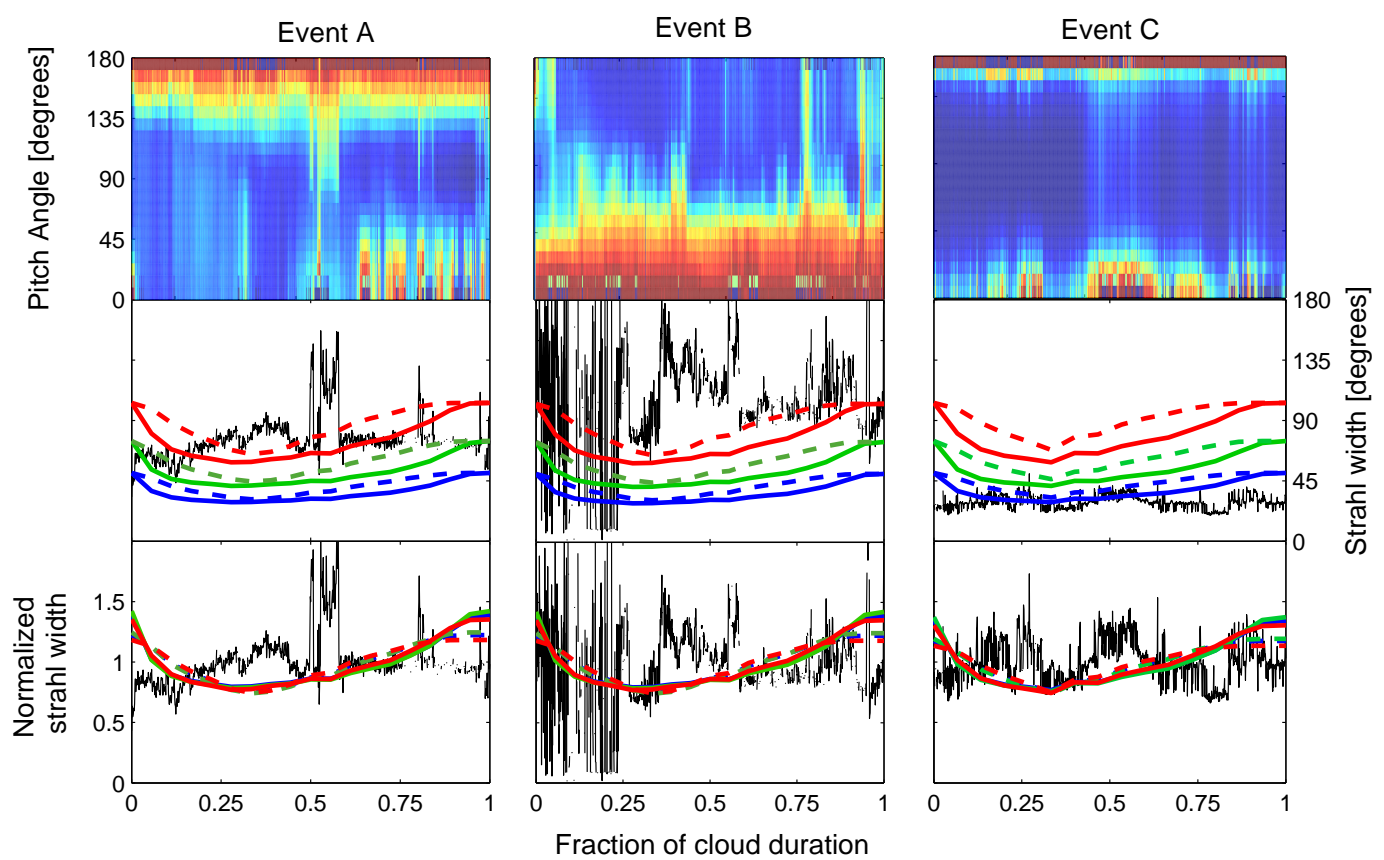

Fig. 7. The suprathermal electron properties inside the three magnetic cloud case studies. The top panels show the normalized pitch-angle distribution within the clouds. The black lines in the middle panels show the widths of the dominant strahls, computed from a Gaussian fit in pitch-angle space, with the colored lines showing the expected strahl width variations for different flux rope encounters (in the same format as Fig. 4). The bottom panels show the normalized strahl widths in the same format.
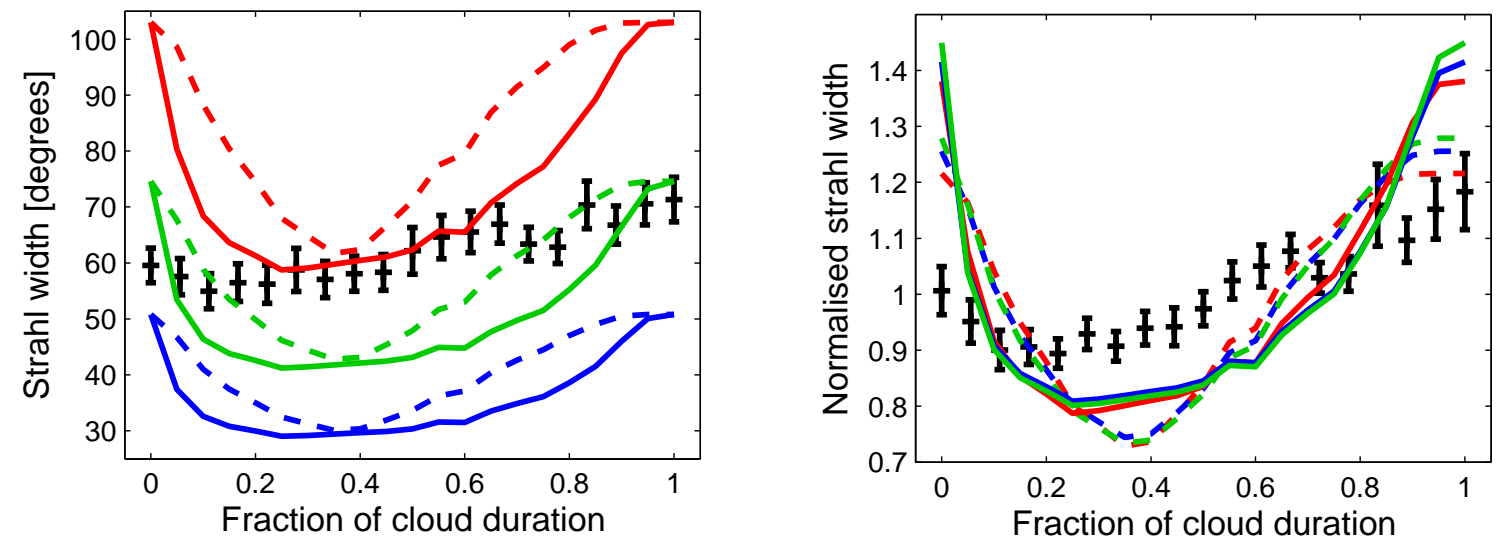

Fig. 8. A superposed epoch plot of the strahl widths in all 74 magnetic clouds catalogued by Cane and Richardson (2003) between 1998 and 2007. Coloured lines show the model predictions in the same format as Fig. 4. In the right panel there is some evidence of the predicted trend in the normalized strahl width observations.

the ambient solar wind imposes a maximum electron time of flight of $1 \mathrm{AU} / V_{S W}$, independent of field-line length.

The expected variation in strahl width is found to be dependent on the level of scattering in the sense that magnetic clouds with broader strahls throughout should exhibit a more pronounced flux rope imprint. If, however, the strahl width is normalised to the average strahl width observed in the event, the flux rope signature is independent of the scattering rate.
For the flux rope signature to be observable, the maximum change in strahl width through a flux rope, $\triangle \mathrm{FWHM}$, must be above the pitch-angle resolution of the instrument used to measure the suprathermal electron distribution. For the standard ACE SWEPAM data set (McComas et al., 1998), this cut-off point is $9^{\circ}$. Even for magnetic cloud encounters far from the axis of the flux rope, this signature should, in principle, be observable for a range of scattering rates. 
Suprathermal electron observations for three classic examples of magnetic clouds were examined. There was large event-to-event variation in strahl widths, but none of the events exhibited the expected flux rope signature. We also performed an statistical survey of 74 magnetic clouds. Although the superposed epoch analysis performed here removes much of the information about event-to-event variability, there is nevertheless some evidence of the expected strahl width variation, though it is a much weaker signature than the models predict. This aspect of the study clearly merits further, more detailed, investigation.

Possible reasons why the observed strahl width variation is weaker than predicted can be broadly categorized into unmet assumptions about the magnetic field structure of magnetic clouds and the suprathermal electron scattering.

Assuming first that the field-line length has been accurately determined, it is necessary to consider the assumptions made in determining the associated strahl width at $1 \mathrm{AU}$. The model of Owens et al. (2008) assumes that all scattering undergone by suprathermal electrons occurs in pitch-angle space, and electrons do not lose or gain energy. There is support for this assumption in the observed conservation of electrons scattered from the strahl to the halo at a given energy (Maksimovic et al., 2005), but the postulated energy loss by cross-field drift in the motional electric field has yet to be evaluated (J. R. Jokipii and N. A. Schwadron, personal communication, 2008, 2009). We are also assuming that there is no systematic variation in the scattering properties within a MFR by applying the same scattering rate throughout the structure. If scattering is enhanced at the axis of a flux rope and reduces toward the outer edge, it will act against the field-line length variation and reduce the flux rope signature on suprathermal electrons. There is no reason to expect this behavior, but we cannot discount the possibility.

The most fundamental assumption we have made about the structure of a magnetic cloud is that, locally at least, it forms a flux rope with helical fields that increase in pitch from the straight central axis to the tightly wound edge. This is the widely accepted explanation for the observed magnetic field rotation and is thus unlikely to be an unmet assumption responsible for the weak flux rope imprint, however, there has been recent speculation that a field rotation may not always indicate a flux rope structure (Jacobs et al., 2009).

Setting $\alpha$ equal to 2.408 , which implicitly assumes the flux rope edge to be occur where the field becomes entirely poloidal, also has implications for the findings in this study. If the flux rope field is not so tightly wound, or the outer, tightest-wound fields are removed by magnetic reconnection with the ambient solar wind (Schmidt and Cargill, 2003), then the expected variation in field-line length, and hence strahl width, will be reduced. The fact that the field in the maximum variance direction is often seen to rotate through a full $180^{\circ}$, however, as in Event A, argues in favor of our assumption about $\alpha$.
Magnetic reconnection may be at least a partial explanation for the weak flux rope signature in suprathermal electrons. Near the Sun reconnection is known to open up the closed loops within magnetic clouds (Crooker and Webb, 2006). This process will move the flux rope foot points about at the photosphere but is unlikely to significantly affect the field-line length. Magnetic reconnection between different flux tubes within the magnetic cloud (Gosling et al., 2007), however, would serve to mix field lines of different length and possibly reduce the suprathermal electron signature.

A second possibility is a systematic increase in adiabatic focusing with distance from the magnetic cloud axis, counteracting the extra scattering time from increased field line length. The peak magnetic field intensity is often located close to the centre of a magnetic cloud, so if the foot point field strength back at the Sun is uniform, this suggests that there is a greater variation in field strength between the Sun and $1 \mathrm{AU}$ close to the edges of the flux rope, and hence a stronger focusing effect. Further observational analysis and modelling efforts are required to establish the significance of this effect.

Acknowledgements. Research at Imperial College London is funded by STFC (UK), NC is funded by NSF grant ATM-0553397. We have benefited from the availability of ACE magnetic field (PI: C. Smith) and SWEPAM (PI: D. McComas) data. MO thanks Benoit Lavraud of CESR (Toulouse), for useful discussions.

Editor in Chief W. Kofman thanks S. Kahler and another anonymous referee for their help in evaluating this paper.

\section{References}

Anderson, B. R., Skoug, R. M., Steinberg, J. T., and McComas, D. J.: Comparison of the Width and Intensity of the Suprathermal Electron Strahl in General Solar Wind and ICME Solar Wind., AGU Fall Meeting Abstracts, pp. A1580+, 2008.

Bothmer, V. and Schwenn, R.: The structure and origin of magnetic clouds in the solar wind, Ann. Geophys., 16, 1-24, 1998, http://www.ann-geophys.net/16/1/1998/.

Burlaga, L. F.: Magnetic clouds: Constant alpha force-free configurations, J. Geophys. Res., 93, 7217-7224, 1988.

Burlaga, L. F., Sittler, E., Mariani, F., and Schwenn, R.: Magnetic loop behind and interplanetary shock: Voyager, Helios, and IMP 8 observations, J. Geophys. Res., 86, 6673-6684, 1981.

Cane, H. V. and Richardson, I. G.: Interplanetary coronal mass ejections in the near-Earth solar wind during 1996-2002, J. Geophys. Res., 108, A41156, doi:10.1029/2002JA009817, 2003.

Chollet, E. E., Giacalone, J., Mazur, J. E., and Al Dayeh, M.: A New Phenomenon in Impulsive-Flare-Associated Energetic Particles, Astrophys. J., 669, 615-620, doi:10.1086/521670, 2007.

Crooker, N. U. and Webb, D. F.: Remote sensing of the solar site of interchange reconnection associated with the May 1997 magnetic cloud, J. Geophys. Res., 111, A08108, doi:10.1029/ 2006JA011649, 2006.

Crooker, N. U., Kahler, S. W., Gosling, J. T., and Lepping, R. P.: Evidence in magnetic clouds for systematic open flux trans- 
port on the Sun, J. Geophys. Res., 113, 12107, doi:10.1029/ 2008JA013628, 2008.

Feldman, W. C., Asbridge, J. R., Bame, S. J., Montgomery, M. D., and Gary, S. P.: Solar wind electrons, J. Geophys. Res., 80, 4181-4196, 1975.

Gosling, J. T., Baker, D. N., Bame, S. J., Feldman, W. C., and Zwickl, R. D.: Bidirectional solar wind electron heat flux events, J. Geophys. Res., 92, 8519-8535, 1987.

Gosling, J. T., Eriksson, S., McComas, D. J., Phan, T. D., and Skoug, R. M.: Multiple magnetic reconnection sites associated with a coronal mass ejection in the solar wind, J. Geophys. Res., 112, 8106, doi:10.1029/2007JA012418, 2007.

Hammond, C. M., Feldman, W. C., McComas, D. J., Phillips, J. L., and Forsyth, R. J.: Variation of electron-strahl width in the highspeed solar wind: ULYSSES observations, Astron. Astrophys., 316, 350-354, 1996.

Jacobs, C., Roussev, I. I., Lugaz, N., and Poedts, S.: The Internal Structure of Coronal Mass Ejections: Are all Regular Magnetic Clouds Flux Ropes?, Astrophys. J. Lett., 695, L171-L175, doi: 10.1088/0004-637X/695/2/L171, 2009.

Larson, D. E., Lin, R. P., McTiernan, J. M., McFadden, J. P., Ergun, R. E., McCarthy, M., Rème, H., Sanderson, T. R., Kaiser, M., Lepping, R. P., and Mazur, J.: Tracing the topology of the October 18-20, 1995, magnetic cloud with $\sim 0.1-10^{2} \mathrm{keV}$ electrons, Geophys. Res. Lett., 24, 1911-1914, doi:10.1029/97GL01878, 1997.

Lepping, R. P., Jones, J. A., and Burlaga, L. F.: Magnetic field structure of interplanetary clouds at 1 AU, J. Geophys. Res., 95, 11957-11965, 1990.

Lundquist, S.: Magnetostatic fields, Arkiv foer Fysik, 2, 361-365, 1950.

Maksimovic, M., Zouganelis, I., Chaufray, J.-Y., Issautier, K., Scime, E. E., Littleton, J. E., Marsch, E., McComas, D. J., Salem, C., Lin, R. P., and Elliott, H.: Radial evolution of the electron distribution functions in the fast solar wind between 0.3 and $1.5 \mathrm{AU}$, J. Geophys. Res., 110, A09104, doi:10.1029/2005JA011119, 2005.

McComas, D. J., Gosling, J. T., Winterhalter, D., and Smith, E. J.: Interplanetary magnetic field draping about fast coronal mass ejecta in the outer heliosphere, J. Geophys. Res., 93, 2519-2526, doi:10.1029/JA093iA04p02519, 1988.

McComas, D. J., Bame, S. J., J., B. S., Feldman, W. C., Phillips, J. L., Riley, P., and Griffee, J. W.: Solar wind electron proton alpha monitor (SWEPAM) for the Advanced Composition Explorer, Space Sci. Rev., 86, 563, 1998.
Newkirk, Jr., G., Hundhausen, A. J., and Pizzo, V.: Solar cycle modulation of galactic cosmic rays - Speculation on the role of coronal transients, J. Geophys. Res., 86, 5387-5396, 1981.

Owens, M. J.: Magnetic cloud distortion resulting from propagation through a structured solar wind: Models and observations, J. Geophys. Res., 111, A12109, doi:10.1029/2006JA011903, 2006.

Owens, M. J. and Crooker, N. U.: Coronal mass ejections and magnetic flux buildup in the heliosphere, J. Geophys. Res., 111, A10104, doi:10.1029/2006JA011641, 2006.

Owens, M. J. and Crooker, N. U.: Reconciling the electron counterstreaming and dropout occurrence rates with the heliospheric flux budget, J. Geophys. Res., 112, A06106, doi:10.1029/ 2006JA012159, 2007.

Owens, M. J., Cargill, P. J., Pagel, C., Siscoe, G. L., and Crooker, N. U.: Characteristic magnetic field and speed properties of interplanetary coronal mass ejections and their sheath regions, J. Geophys. Res., 110, A01105, doi:10.1029/2004JA010814, 2005.

Owens, M. J., Merkin, V. G., and Riley, P.: A kinematically distorted flux rope model for magnetic clouds, J. Geophys. Res. 111, A03104, doi:10.1029/2005JA011460, 2006.

Owens, M. J., Crooker, N. U., and Schwadron, N. A.: Suprathermal electron evolution in a Parker spiral magnetic field, J. Geophys. Res., 113, A11104, doi:10.1029/2008JA013294, 2008.

Pilipp, W. G., Muehlhaeuser, K.-H., Miggenrieder, H., Rosenbauer, H., and Schwenn, R.: Variations of electron distribution functions in the solar wind, J. Geophys. Res., 92, 1103-1118, 1987.

Riley, P. and Crooker, N. U.: Kinematic treatment of CME evolution in the solar wind, Astrophys. J., 600, 1035-1042, 2004.

Rosenbauer, H., Schwenn, R., Marsch, E., Meyer, B., Miggenrieder, H., Montgomery, M. D., Muehlhaeuser, K. H., Pilipp, W., Voges, W., and Zink, S. M.: A survey on initial results of the HELIOS plasma experiment, Journal of Geophysics Zeitschrift Geophysik, 42, 561-580, 1977.

Schmidt, J. M. and Cargill, P. J.: Magnetic reconnection between a magnetic cloud and the solar wind magnetic field, J. Geophys. Res., 108, 1023, doi:10.1029/2002JA009325, 2003.

Wimmer-Schweingruber, R. F., Crooker, N. U., Balogh, A., Bothmer, V., Forsyth, R. J., Gazis, P., Gosling, J. T., Horbury, T., Kilchmann, A., Richardson, I. G., Riley, P., Rodriguez, L., von Steiger, R., Wurz, P., and Zurbuchen, T. H.: Understanding interplanetary coronal mass ejection signatures, Space Sci. Rev., 123 , 177-216, doi:10.1007/s11214-006-9017-x, 2006. 